\title{
ESTRATÉGIAS DE INOVAÇÃO PARA STARTUPS
}

\author{
INNOVATION STRATEGIES TO STARTUPS
}

\author{
Ronalty Oliveira Rocha \\ Mestre em Administração pela Universidade Federal de Sergipe. \\ Universidade Federal de Sergipe, CCSA-Centro de Ciências Sociais Aplicadas, Programa de Pós-graduação em Administração. \\ São Cristóvão, Brasil. \\ Email: mleonolave@gmail.com \\ Maria Elena Leon Olave \\ Doutorado em Engenharia de Produção pela Universidade de São Paulo (2003). \\ Professora Associada do Departamento de Administração da Universidade Federal de Sergipe, Docente do Programa de Pós- \\ Graduação em Administração- PROPADM/UFS e do Programa de Mestrado Profissional em Administração - (PROFIAP). \\ São Cristóvão, SE, Brasil. \\ Email: mleonolave@gmail.com \\ Edward David Moreno Ordonez \\ Doutorado em Engenharia Elétrica pela Universidade de São Paulo (1998). \\ Professor do Departamento de Computação da Universidade Federal de Sergipe. \\ São Cristóvão, SE, Brasil. \\ Email: edwdavid@gmail.com
}

\section{RESUMO}

Não basta apenas que as organizações reconheçam a importância da inovação para o sucesso e sobrevivência de seus negócios, é preciso também que estejam atentas e aptas a seleção e implementação das melhores estratégias de inovação. Neste cenário, em que inovação e organizações empresariais parecem andar juntas, algumas modalidades de negócios se destacam por seu potencial para implementação de inovações, como é o caso das startups. As startups são empresas de base tecnológica, com amplo potencial de crescimento e desenvolvimento de inovações pela inserção de novos produtos e serviços em negócios replicáveis. Em conformidade com o exposto, este artigo, apresentado sob a forma de um ensaio teórico, tem como objetivo propor um modelo teórico que integre seleção de tipologias e estratégias de inovação voltadas a aumentar a prática de inovação em startups de tecnologia da informação. No modelo proposto é apresentada uma interconexão de atividades que revela como a definição de um processo de inovação influencia e é influenciado pela adoção de tipologias e estratégias de inovação. O intuito do modelo proposto é associar às atividades de startups com a definição de tipologias e estratégias de inovação que promovam melhores resultados empresariais, assim como promovam ganhos em aprendizado e práticas de inovação.

Palavras-chave: Inovação. Tipologias de inovação. Estratégias de inovação. Startups. Modelo conceitual

Data de submissão: 19 de março de 2018.

\section{ABSTRACT}

It is not enough that organizations recognize the importance of innovation for the success and survival of their business, it is also necessary that they are attentive and apt the selection and implementation of the best strategies of innovation. In this scenario, where innovation and business organizations seem to go hand in hand, some business modalities stand out because of their potential for implementing innovations, such as startups. Startups are technology-based companies with broad potential for growth and development of innovations by the insertion of new products and services into replicable businesses. In accordance with the above, this article, presented in the form of a theoretical essay, aims to propose a theoretical model that integrates selection of typologies and innovation strategies aimed at increasing the practice of innovation in information technology startups. In the proposed model is presented an interconnection of activities that reveals how the definition of an innovation process influences and is influenced by the adoption of typologies and innovation strategies. The purpose of the proposed model is to associate startup activities with the definition of typologies and innovation strategies that promote better business results, as well as promote gains in learning and innovation practices.

Keywords: Innovation. Innovation typologies. Innovation strategies. Startups. Conceptual model. 


\section{INTRODUÇÃO}

Com a expansão no uso de ferramentas de tecnologia da informação as organizações passaram a lidar com o imperativo da inovação, que tem sido indicada como um dos mais proeminentes meios para manutenção da competitividade empresarial. Inclusive, é consenso entre diferentes estudiosos que a inovação é um dos principais motores para desempenho empresarial e socioeconômico, diferenciação e vantagem competitiva das organizações (STOILOV, 2015; IMBUZEIRO, 2014; CROPLEY; KAUFMAN; CROPLEY, 2011).

Ao considerar o papel da inovação como instrumento para desenvolvimento econômico e crescimento empresarial, é também necessário analisar como as empresas têm se dedicado a prática da inovação, como estratégia. Heidenreich e Kraemer (2016) explicam que as estratégias de inovação se referem à seleção dos melhores e mais adequados planos organizacionais para o desenvolvimento e inserção de novos produtos e serviços, assim como para a abertura de novos mercados.

Nesse contexto, é importante destacar que a literatura acadêmica dispõe de diferentes estratégias para inovação. Cada tipologia de estratégia enfatiza um conjunto distinto de atividades, algumas delas focalizam em parcerias com clientes (VERLEYE, 2015), outras defendem a prática de inovação fechada, exclusivamente no interior das organizações (SCHUMPETER, 1988), ainda existem estratégias que defendem a internacionalização como atividade promotora da inovação (REN; EISINGERICH; TSAI, 2015), ao passo em que outras tipologias de estratégias enfatizam a colaboração com diferentes instituições, como concorrentes, fornecedores e instituições de ensino e pesquisa (CHESBROUGH, 2003, 2012), além das estratégias focadas em parcerias com outras organizações (ALBERTI; PIZZURNO, 2017).

Hunt (2013) cita as empresas startups como promotoras da inovação. Startups, pela definição adotada nesse estudo, são empresas que têm a inovação em seu núcleo (RIES, 2012), são orientadas pela tecnologia da informação e detém grande potencial de crescimento pela inserção de inovações no mercado (ROBEHMED, 2013; SHONTELL, 2014).

Especificamente às startups de tecnologia da informação, Turri e Wagner (2015) apontaram que esses negócios são alavancadores da inovação, posto que inserem novos produtos e serviços, desenvolvem novos processos de produção e novas técnicas organizacionais (FERNANDES, 2015) explorando novos nichos de mercado e atraindo investidores.

Diante do exposto e considerando o potencial de desenvolvimento econômico e geração de empregos em startups, assim como considerando a importância da adequada seleção de estratégias dirigidas para inovação buscando a sobrevivência desses negócios, esse artigo tem como objetivo propor um modelo teórico que integre seleção de tipologias e estratégias de inovação voltadas a aumentar a prática de inovação em startups de tecnologia da informação.

\section{INOVAÇÃO: ASPECTOS CONCEITUAIS}

Inicialmente é necessário apresentar, ainda que brevemente, o conceito de inovação. Sobre esse assunto, Ismail e Abdmajid (2007) lembram-nos que por mais que a inovação tenha alcançado destaque na literatura acadêmica, os estudos sobre esse tema ainda são complexos e fragmentados, uma vez que os diferentes autores que tratam do tema o analisam sob diferentes posições ontológicas e epistemológicas. 
A fim de democratizar debates sobre o tema apresenta-se no Quadro 1 uma breve revisão sobre os conceitos de inovação e os autores que os defendem.

Quadro 1 - Conceitos de inovação

\begin{tabular}{|c|c|c|}
\hline Elemento definidor & Autores & Definição \\
\hline \multirow{3}{*}{ Introdução de novidades } & Schumpeter (1939). & $\begin{array}{l}\text { Novas combinações de recursos já existentes para } \\
\text { acessar novos mercados, produzir novos produtos, ou } \\
\text { produzir produtos antigos de uma forma mais eficiente. }\end{array}$ \\
\hline & $\begin{array}{l}\text { Manual de Oslo (OCDE, } \\
\text { 2006). }\end{array}$ & $\begin{array}{l}\text { Implementação de um novo, ou melhorado, produto ou } \\
\text { serviço, processo produtivo, método de marketing e/ou } \\
\text { método organizacional nas práticas de negócios, } \\
\text { organização no local de trabalho e/ou relações } \\
\text { empresariais. }\end{array}$ \\
\hline & Tidd, Bessant e Pavitt (2008) & $\begin{array}{l}\text { Conjunto de atividades organizacionais para criação ou } \\
\text { aquisição de soluções, prototipação, tentativas, } \\
\text { produção e comercialização de produtos e serviços } \\
\text { inéditos ou melhorados. }\end{array}$ \\
\hline $\begin{array}{l}\text { Introdução de novidades } \\
\text { e agentes participantes }\end{array}$ & $\begin{array}{l}\text { Bledow et al (2009); Cropley, } \\
\text { Kaufman e Cropley (2011) }\end{array}$ & $\begin{array}{l}\text { Desenvolvimento e introdução, intencional, de novas, } \\
\text { úteis e práticas ideias por indivíduos, equipes e } \\
\text { organizações. }\end{array}$ \\
\hline $\begin{array}{l}\text { Aprendizagem } \\
\text { organizacional }\end{array}$ & $\begin{array}{l}\text { Camisón e Monfort-Mir } \\
(2012)\end{array}$ & $\begin{array}{l}\text { Resultado visível da capacidade das organizações de } \\
\text { gerar novos conhecimento e formas de utilização, } \\
\text { combinação, síntese e/ou melhorias substanciais para a } \\
\text { introdução de novidades no mercado. }\end{array}$ \\
\hline $\begin{array}{l}\text { Desempenho } \\
\text { empresarial }\end{array}$ & $\begin{array}{l}\text { Cropley, Kaufman e Cropley } \\
\text { (2011); Ramadani; Gerguri } \\
\text { (2011); Stoilov (2015). }\end{array}$ & $\begin{array}{l}\text { Capacidade das organizações de influenciar seus } \\
\text { recursos organizacionais de marketing, habilidades } \\
\text { gerenciais, conhecimentos, capacidades e estratégias } \\
\text { organizacionais em prol da eficácia organizacional e } \\
\text { sucesso empresarial. }\end{array}$ \\
\hline \multirow{2}{*}{$\begin{array}{l}\text { Desempenho } \\
\text { empresarial e } \\
\text { desenvolvimento } \\
\text { socioeconômico }\end{array}$} & Imbuzeiro (2014). & $\begin{array}{l}\text { Conversão de uma nova ideia em algo que possa ser } \\
\text { comercializável ou proveitoso ao progresso das } \\
\text { empresas, regiões e economias. }\end{array}$ \\
\hline & $\begin{array}{l}\text { Huarng; Ribeiro-Soriano, } \\
\text { (2014); Mas-tur et al., } \\
\text { (2015). }\end{array}$ & $\begin{array}{l}\text { Além de fomentar a criação de produtos e processos, a } \\
\text { inovação favorece o desempenho de negócios, bem- } \\
\text { estar social e criação de riquezas em uma região ou } \\
\text { país. }\end{array}$ \\
\hline
\end{tabular}

Fonte: Elaborado pelos autores (2018)

Conforme pode ser verificado o conceito de inovação envolve diferentes aspectos, mas todos eles convergem com a ideia de que a inovação representa a inserção e comercialização de algo novo, seja um produto, um processo, um método organizacional ou uma prática de marketing, sendo estas, inclusive, as principais tipologias de inovação (OCDE, 2006).

É pertinente explicar que as inovações de produto, de serviço e de processo são, genericamente, denominadas de inovações tecnológicas, isto porque utilizam recursos baseados nos conhecimentos científicos e tecnológicos desenvolvidos em universidades, institutos de pesquisa ou nas próprias empresas 
(SILVA; WESCHENFELDER; ESTEVES, 2014). D'alvano e Hidalgo (2012) complementam que as inovações de produto, serviço e processo são de caráter tecnológico por estarem inseridas num conjunto de etapas técnicas, industriais e comerciais que sustentam o lançamento bem-sucedido de novos produtos e serviços no mercado comercial ou a utilização comercial de novos processos técnicos. No Quadro 2 são brevemente descritas as principais tipologias de inovação.

Quadro 2 - Tipologias de inovação

\begin{tabular}{|c|c|c|}
\hline Autor & Tipologia & Descrição \\
\hline \multirow[b]{2}{*}{$\begin{array}{l}\text { Schumpeter (1988); OCDE } \\
\text { (2006); Akcigit (2010); } \\
\text { Imbuzeiro (2014); Koc e } \\
\text { Bozdag (2017). }\end{array}$} & Inovação radical & \multirow[b]{2}{*}{$\begin{array}{l}\text { Inovações radicais produzem mudanças } \\
\text { fundamentais nas atividades de uma organização } \\
\text { e grandes desvios nas práticas existentes, ao } \\
\text { passo que as inovações incrementais } \\
\text { representam um menor grau de desvio das atuais } \\
\text { práticas de uma organização. }\end{array}$} \\
\hline & Inovação incremental & \\
\hline $\begin{array}{l}\text { Christensen, Johnson e Rigby } \\
\text { (2002); Besanko et al., (2010). }\end{array}$ & Inovação disruptiva & $\begin{array}{l}\text { Inovações que permitem a entrada de novos } \\
\text { participantes no mercado, a partir de soluções } \\
\text { simples, rápido crescimento mercadológico. }\end{array}$ \\
\hline $\begin{array}{l}\text { Schumpeter (1988); OCDE } \\
\text { (2006); Silva, Weschenfelder e } \\
\text { Esteves (2014); Heidenreich e } \\
\text { Kraemer (2016); Jacobs et al., } \\
\text { (2016). }\end{array}$ & Inovação de produto/Serviço & $\begin{array}{l}\text { Introdução de produtos/serviços novos ou } \\
\text { significativamente melhorados em termos de suas } \\
\text { características ou utilização pretendida }\end{array}$ \\
\hline $\begin{array}{l}\text { OCDE (2006); Tidd, Bessant e } \\
\text { Pavitt (2008); Forsman (2011); } \\
\text { Ruzzier, Hojnik e Lipnik (2013); } \\
\text { Imbuzeiro (2014). }\end{array}$ & Inovação de processos & $\begin{array}{l}\text { Referem-se às mudanças na forma em que os } \\
\text { produtos ou serviços são criados e entregues }\end{array}$ \\
\hline $\begin{array}{l}\text { Kimberly (1981); Evan (1996); } \\
\text { Aravind (2012); Ayhan e } \\
\text { Oztemel (2014); Hamidi e } \\
\text { Benabdeljlil, (2015); Jacobs et } \\
\text { al., (2016). }\end{array}$ & $\begin{array}{l}\text { Inovação } \\
\text { organizacional/Gerencial/ } \\
\text { Administrativa }\end{array}$ & $\begin{array}{l}\text { Criação e implementação de novas práticas, } \\
\text { processos, estruturas e/ou técnicas de gestão, } \\
\text { que contribuem para maior organização, } \\
\text { eficiência e desempenho dos negócios. }\end{array}$ \\
\hline $\begin{array}{l}\text { OCDE (2006); Takahashi } \\
\text { (2012); Imbuzeiro (2014). }\end{array}$ & Inovação de Marketing & $\begin{array}{l}\text { Mudanças na concepção e promoção do produto, } \\
\text { seja por alterações significativas no desenho ou } \\
\text { embalagem, posicionamento, alcance de novos } \\
\text { mercados, promoção e preço. }\end{array}$ \\
\hline Tidd, Bessant e Pavitt (2008). & Inovação de Posição & $\begin{array}{l}\text { Introdução de mudanças no contexto em que } \\
\text { produtos ou serviços são lançados no mercado. }\end{array}$ \\
\hline Tidd, Bessant e Pavitt (2008). & Inovação de Paradigma & $\begin{array}{l}\text { Mudanças nos modelos mentais implícitos que } \\
\text { orientam o que a empresa faz. }\end{array}$ \\
\hline $\begin{array}{l}\text { Agarwal e Brem (2012); Radjou } \\
\text { e Prabhu (2013); Tiwari; } \\
\text { Kalogerakis; Herstatt (2014). }\end{array}$ & Inovação Frugal & $\begin{array}{l}\text { Produzir, redesenhar e desenvolver produtos e } \\
\text { processos a um custo mínimo. }\end{array}$ \\
\hline
\end{tabular}

Fonte: Elaborado pelos autores (2018) 
É importante deixar claro que uma inovação pode se enquadrar em diferentes tipologias, inclusive há a possibilidade de combinação de diferentes tipos de inovação o que provoca nas organizações o desenvolvimento de diferentes capacidades, assim como favorece ao alcance de objetivos empresariais (DAMANPOUR; WALKER; AVELLANEDA, 2009).

Apresentado o conceito e as tipologias de inovação, faz-se necessário também debater as estratégias de inovação consideradas pelas organizações, especialmente por empresas startups, objeto de estudo nesse artigo.

\section{ESTRATÉGIAS DE INOVAÇÃO}

A inovação representa um imperativo estratégico nas economias de hoje (HEIDENREICH; KRAEMER, 2016), e as estratégias para inovação referem-se à seleção dos melhores e mais adequados planos organizacionais para o desenvolvimento e inserção de novos produtos e serviços, assim como para a entrada de novos mercados (SANCHES; MACHADO, 2014).

Logo, as estratégias para inovação são abordagens organizacionais para definição dos objetivos e escolha de métodos que serão utilizados para potencializar resultados através de inovações (LENDEL; VARMUS, 2011). Sobre esse tema, Barbosa e Machado (2013) comentam que as estratégias de inovação estão distribuídas em diversas classificações, uma vez que cada autor considera um conjunto distinto de aspectos e parâmetros de tipificação.

É importante apontar que a literatura acadêmica apresenta diversas estratégias de inovação, tais como: Ser proativa e ser reativa (GILBERT, 1997); estratégia de processo, estratégia de velocidade, estratégia de inovação por aprendizagem, estratégia de mercado, estratégia de tecnologia, estratégia de inovação quantitativa (LYNN; AKGUN, 1998); estratégia jogar para ganhar, estratégia jogar para não perder (DÁVILA; EPSTEIN; SHELTON, 2007); estratégia ofensiva, estratégia defensiva, estratégias imitativas, estratégia dependente, estratégia tradicional, estratégia oportunista (FREEMAN; SOETE, 2008); estratégia Explorative, estratégia Exploitative (FAUCHART; KEILBACH, 2009) e inovação fechada (SCHUMPETER, 1988); LOVE; ROPER (2015); HSIEH; LEE; HUANG, 2016).

Contudo, Doloreux (2013) argumenta que em startups o processo de inovação acontece com o auxilio de múltiplas funções, atores e recursos dentro e entre fronteiras das empresas que formam um conjunto expandido de competências divergentes e que são, fundamentalmente, necessárias para transformar ideias inovadoras em inovações que se mostrem economicamente bem-sucedidas (BERNE 2016).

A partir do entendimento desses autores, especialmente no tocante as competências divergentes entre as fronteiras organizacionais que despertam o processo inovador em startups, esse trabalho preferiu, então, focar nas estratégias de parcerias com clientes, parcerias com outras organizações, inovação aberta e internacionalização como estratégias prováveis a startups, conforme é apresentado no Quadro 3. 
Quadro 3 - Estratégias de inovação

\begin{tabular}{|c|c|c|}
\hline Autores & Estratégia & Esclarecimento \\
\hline $\begin{array}{l}\text { Drechsler e Natter (2012); } \\
\text { Saebi e Foss (2014); } \\
\text { Alberti e Pizzurno (2017). }\end{array}$ & Parcerias com outras organizações & $\begin{array}{l}\text { As organizações buscam } \\
\text { conhecimentos externos, por meio de } \\
\text { parcerias com outras organizações, } \\
\text { para adquirir expertises, avaliar } \\
\text { contextos empresariais e incrementar } \\
\text { a carteira interna de soluções. }\end{array}$ \\
\hline $\begin{array}{l}\text { Greer e Lei (2012); Seran e } \\
\text { Izvercian (2014); Melo et al., } \\
\text { (2015); Verleye (2015). }\end{array}$ & $\begin{array}{l}\text { Parcerias com clientes; co-criação; co- } \\
\text { desenvolvimento; co-inovação; } \\
\text { coprodução; inovação colaborativa; } \\
\text { usuários líderes; inovação participativa } \\
\text { e/ou inovação centrada no usuário. }\end{array}$ & $\begin{array}{l}\text { Esforço conjunto entre empresa e } \\
\text { clientes para o desenvolvimento de } \\
\text { novos produtos e serviços por meio da } \\
\text { comunicação e interação. }\end{array}$ \\
\hline $\begin{array}{l}\text { Chesbrough (2003, 2012); } \\
\text { Mas-tur et al., (2015); Saebi e } \\
\text { Foss (2014); Hsieh, Lee e } \\
\text { Huang (2016); Varichio (2016). }\end{array}$ & Inovação Aberta & $\begin{array}{l}\text { Busca externa de conhecimentos e } \\
\text { colaboração com clientes, } \\
\text { fornecedores, concorrentes e } \\
\text { instituições de pesquisa com o intuito } \\
\text { de combinar ideias internas com } \\
\text { tecnologias externas para alavancar o } \\
\text { potencial de novidades em novos } \\
\text { mercados. }\end{array}$ \\
\hline $\begin{array}{l}\text { García, Avella e Ferna'ndez } \\
\text { (2012); Ren, Eisingerich e Tsai } \\
\text { (2015). }\end{array}$ & Internacionalização & $\begin{array}{l}\text { Empresas exportadoras podem } \\
\text { aprender com seus contatos } \\
\text { estrangeiros, adotar novas tecnologias } \\
\text { de produção e, consequentemente, } \\
\text { aumentar a produtividade e } \\
\text { desempenho organizacional. }\end{array}$ \\
\hline
\end{tabular}

Fonte: Elaborado pelos autores (2018)

Apresentadas as principais estratégias para inovação e em conformidade com Barbosa e Machado (2013) percebe-se que não existe estratégia melhor ou pior, há estratégias de natureza diferenciadas, as quais são identificadas e implementadas a partir do plano estratégico global de cada organização, uma vez que, independentemente da tipologia, as estratégias de inovação são adotadas com o objetivo de aumentar a performance, em inovação, da organização ou reduzir lacunas no desempenho que podem surgir conforme as mudanças no ambiente empresarial (CARPEJANI, 2015).

Conforme mencionado, anteriormente, algumas empresas se destacam pelo amplo potencial para inserção de novidades no mercado, a exemplo de empresas startups. Empresas startups possuem reconhecido potencial para inserção de inovações no mercado, principalmente as startups de tecnologia da informação, que nos últimos anos, foram as principais responsáveis pela solução de problemas lançados no mercado, e inserção de inovações e soluções para atender e criar as mais diversas necessidades dos consumidores (SEBRAE, 2015). Dada a relevância desses negócios para promoção da inovação, esse assunto será tratado no tópico a seguir. 


\section{INOVAÇÃO EM STARTUPS}

Inicialmente discute-se que a literatura dispõe de vasta conceituação e diferentes pontos de vista acerca do conceito de startups, inclusive o termo tem sido utilizado de maneira ampla e não muito clara para diferentes contextos (MIRANDA; SANTOS JÚNIOR; DIAS, 2016).

Em razão das diferentes abordagens identificadas sobre o conceito de startups, a definição adotada nesse estudo considera os aspectos propostos por Luger e Koo (2005), assim como também as definições propostas por Stoilov (2015) e Alberti e Pizzurno (2017). Para Stoilov (2015) as startups são empresas projetadas para crescer rapidamente, não é necessário trabalhar em tecnologia ou desenvolver inovações de base tecnológica, mas sim usar a tecnologia como uma ferramenta capaz de potencializar o crescimento, replicação e escala desses negócios.

Diante das diferentes definições para startup, esse trabalho entendeu que o termo está startup está vinculado às empresas que têm a inovação em seu núcleo (RIES, 2012), são orientadas pela tecnologia da informação e detém grande potencial de crescimento (ROBEHMED, 2013; SHONTELL, 2014).

Corroborando esse entendimento, Hunt (2013) argumenta que empresas startups desempenham um papel crucial nos processos de inovação, pois no início de suas atividades, na chamada fase de arranque, novas ideias são introduzidas no mercado e transformadas em estratégias economicamente sustentáveis. Aliás, a capacidade inovadora dessas empresas é tão expressiva que esses negócios são apontados como elementos de base para geração de novas ideias para produtos e serviços que irão, rapidamente, responder as demandas do mercado (ANDRADE; LINS FILHO; SILVA, 2016).

Sobre esse assunto, Andrade, Lins Filho e Silva (2016) evidenciam que as startups possuem características especiais para aprendizado organizacional e consequente incremento a processos de inovação. Os autores enfatizam que em startups, a capacidade de aprendizado está ligada a capacidade de inovar desses negócios, principalmente, em razão das startups possuírem características direcionadas ao uso de novos modelos de compartilhamento de conhecimento e comunicação entre funcionários, clientes e parceiros.

Conforme apresentado a inovação apresenta reconhecido potencial para o desenvolvimento e crescimento empresarial das empresas, inclusive das startups. Partindo dessa constatação foram desenvolvidas, suportadas em postulados teóricos, seis proposições de pesquisa, apresentadas no Quadro 4. 
Quadro 4 - Proposições de Pesquisa

\begin{tabular}{|c|c|}
\hline PROPOSIÇÕES & AUTORES \\
\hline $\begin{array}{l}\text { P1. Startups que baseiam suas estratégias de negócios pela } \\
\text { implementação de inovações incrementais baseadas na imitação e } \\
\text { aperfeiçoamento de produtos existentes garantem regularidade de } \\
\text { vendas e sobrevivência empresarial. } \\
\text { P2. Empresas startups que implementam práticas estratégicas para } \\
\text { inovação pelo uso de laboratórios internos de pesquisa } \\
\text { especializados na produção de novos conhecimentos e propriedade } \\
\text { intelectual para exploração comercial atingem maior grau de inovação } \\
\text { e sobrevivência no mercado. }\end{array}$ & $\begin{array}{l}\text { Chesbrough (2012); Love e Roper (2015); } \\
\text { Stoilov (2015); Hsieh, Lee e Huang (2016). }\end{array}$ \\
\hline $\begin{array}{l}\text { P3. O desenvolvimento de colaborações interorganizacionais } \\
\text { baseadas na busca de conhecimentos externos, por meio de } \\
\text { parcerias com outras organizações, para adquirir expertises e } \\
\text { incrementar a carteira interna de soluções está associado a um maior } \\
\text { grau de inovação por startups no mercado. }\end{array}$ & $\begin{array}{l}\text { Forsman (2011); Drechsler e Natter (2012); } \\
\text { Saebi e Foss (2014); Alberti e Pizzurno } \\
(2017) \text {. }\end{array}$ \\
\hline $\begin{array}{l}\text { P4. A Busca externa de conhecimentos e colaboração com clientes, } \\
\text { fornecedores, concorrentes e instituições de pesquisa com o intuito } \\
\text { de combinar ideias internas com tecnologias externas alavanca o } \\
\text { potencial de introdução de novidades por startups e pode torná-las } \\
\text { inovadoras sistêmicas. }\end{array}$ & $\begin{array}{l}\text { Mas-tur et al., (2015); Saebi e Foss (2014); } \\
\text { Agrawal, Hasija e Bhattacharya (2016); } \\
\text { Hsieh, Lee e Huang (2016); Varichio (2016); } \\
\text { Pérez-Luno et al., (2011). }\end{array}$ \\
\hline $\begin{array}{l}\text { P5. A realização de parcerias com clientes promove o } \\
\text { desenvolvimento de novos produtos e serviços, assim como o } \\
\text { aprimoramento dos produtos/serviços existentes, por meio da } \\
\text { comunicação e interação nas startups, e consequentemente, garante } \\
\text { maior nível de inovação e sobrevivência empresarial a essas } \\
\text { empresas. }\end{array}$ & $\begin{array}{l}\text { Belkahla e Triki (2011); Brunswicker e } \\
\text { Vanhaverbeke (2015); Greer e Lei (2012); } \\
\text { Seran e Izvercian (2014); Melo et al., (2015); } \\
\text { Verleye (2015). }\end{array}$ \\
\hline $\begin{array}{l}\text { P6. Startups exportadoras podem aprender com seus contatos } \\
\text { estrangeiros, adotar novas tecnologias de produção e, } \\
\text { consequentemente, aumentar a produtividade, prática de inovação e } \\
\text { desempenho organizacional. }\end{array}$ & $\begin{array}{l}\text { Charles e David (2012); García, Avella e } \\
\text { Ferna'ndez (2012); Ren, Eisingerich e Tsai } \\
(2015) \text {. }\end{array}$ \\
\hline
\end{tabular}

Fonte: Elaborado pelos autores (2018) 


\section{PROPOSTA DE MODELO CONCEITUAL PARA SELEÇÃO E IMPLEMENTAÇÃO DE TIPOLOGIAS E ESTRATÉGIAS DE INOVAÇÃO EM STARTUPS}

Considerando o potencial das startups de tecnologia da informação para inserção de inovações no mercado e subsidiado nas proposições de pesquisa apresentadas foi desenvolvida uma proposta de modelo conceitual, que contempla tipologias e estratégias, para integrar o papel de startups como promotoras da inovação.

Figura 1 - Seleção e Implementação de Tipologias e Estratégias de Inovação em Startups

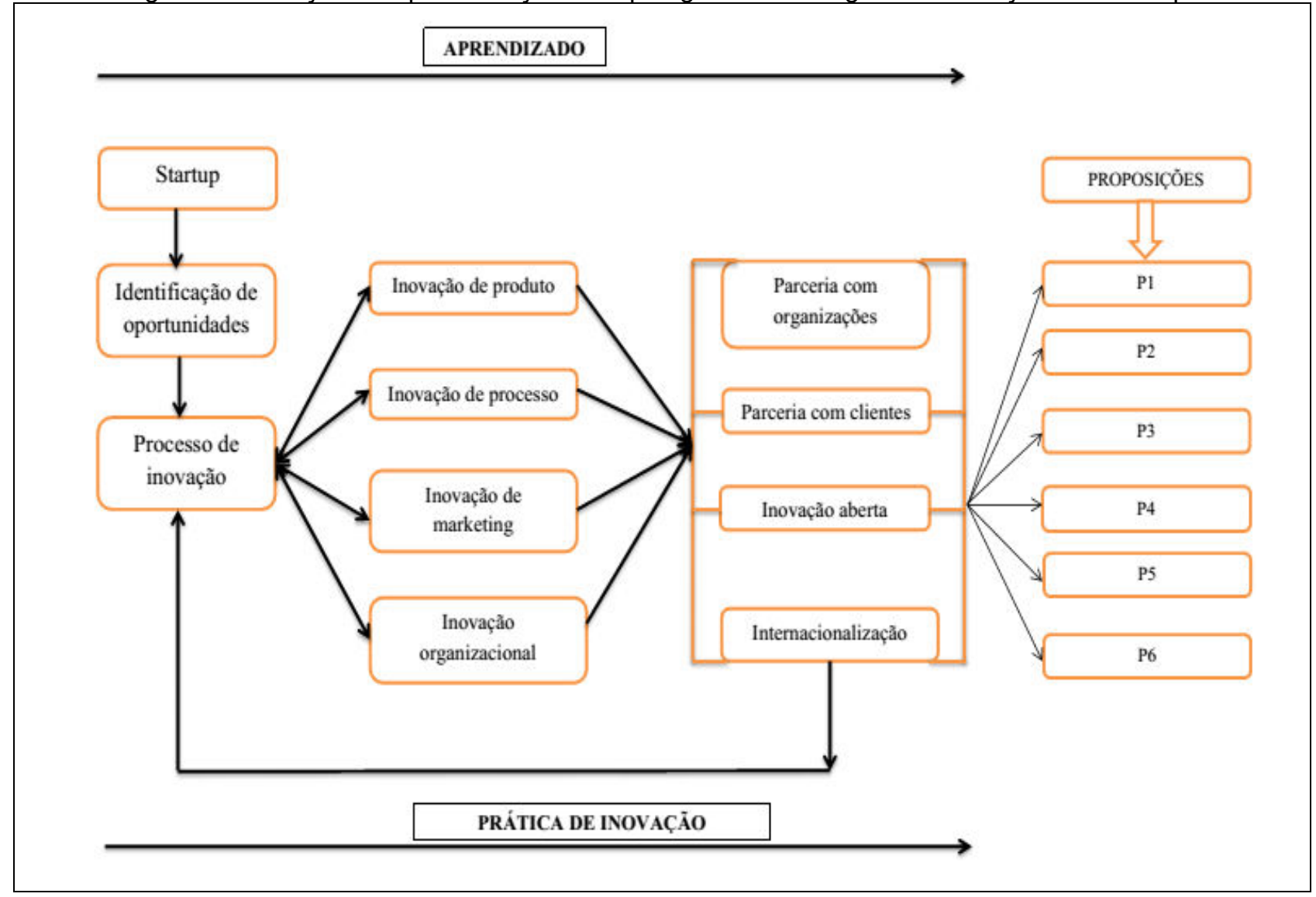

Fonte: Elaborada pelos autores (2018)

No modelo proposto o processo de inovação é iniciado a partir da identificação de alguma oportunidade comercial que instigue a inserção de inovações por startups. Explica-se que no modelo proposto, o processo de inovação consiste no conjunto de procedimentos operacionais e gerenciais que favorece a implementação de determinada inovação, esse processo não, necessariamente, necessita estar estruturado em etapas sequenciais, mas sim adequado a realidade da startup que o implementa, já que o processo de inovação não é uma fórmula pronta, ele é naturalmente estruturado pelos atores incluídos e/ou interessados na sua geração (GOLLO, 2006).

Após a identificação de determinada oportunidade comercial são definidas as tipologias de inovação que favorecem a inserção da inovação projetada. Conforme apresentado no modelo essas inovações podem ter aspectos relacionados a introdução ou melhorias de produtos, processos, práticas de marketing e/ou técnicas de gestão. É importante frisar que as tipologias de inovação carregam aspectos distintos e que uma determinada novidade pode desencadear diferentes tipologias de inovação. A interpretação subjacente é que uma determinada tipologia de inovação irá prevalecer sobre as outras, o que não impede a implementação 
das demais. Assim como o processo de inovação, as tipologias também são influenciadas pela oportunidade identificada e por essa razão influenciam e são implementadas de acordo com o processo de inovação praticado, isto é, o processo de inovação direciona a forma como inovações serão implementadas e as tipologias de inovação consideradas podem também promover ajustes no processo, sendo por razão que as tipologias e processo de inovação estão conectados, no modelo, por setas de duplo sentido.

Implementadas as tipologias mais adequadas ao negócio, percebe-se em quais atividades do processo produtivo e/ou gerencial são necessários ajustes e melhorias, sendo nesse momento que as startups consideram as potenciais estratégias para adquirir novos conhecimentos e incrementar a carteira interna de soluções (PARK; SUBRAMANIAM; STYLIANOU, 2015). Dentre as estratégias apresentadas na revisão de literatura foram selecionadas as estratégias de parceria com organizações (SAEBI; FOSS, 2014), parceria com clientes (VERLEYE, 2015), inovação aberta (CHESBROUGH, 2012) e internacionalização (REN; EISINGERICH; TSAI, 2015). Após a definição e implementação da estratégia de inovação que melhor se adequa a necessidade da startup, podendo inclusive ser mais de uma dentre as estratégias propostas, o processo se reinicia (atualiza), pois o aprendizado e técnicas assimiladas passam a incrementar o conjunto interno de conhecimentos da startup e consequentemente insere mudanças no processo de inovação.

Conforme é demonstrado no modelo, desde a identificação de oportunidades até a definição e prática de tipologias e estratégias, a startup acumula conhecimentos resultantes das etapas implementadas, assim como aumenta seu grau de inovação por inserir práticas que alteram, ajustam e promovem melhorias em seu funcionamento e posicionamento de mercado.

\section{CONCLUSÕES}

A inovação ainda figura entre os principais atributos para sobrevivência e melhor desempenho empresarial. As empresas startups estão atentas a esta realidade e têm buscado inserir inovações disruptivas e incrementais no mercado a fim de alcançar maior número de clientes e lograr maior êxito no mercado empresarial.

Por esta razão este artigo propôs um modelo conceitual com a intenção de integrar a ação de startups com a definição de tipologias e estratégias de inovação que promovam melhores resultados empresariais e em inovação, assim como promovam ganhos em aprendizado e práticas de inovação.

Por se tratar de um ensaio teórico, a validação desse estudo carece de etapas empíricas, dessa forma se faz necessário a replicação dessa pesquisa intentando verificar se a definição de estratégias e tipologias de inovação ocorrem como apresentado no modelo proposto. 


\section{REFERÊNCIAS}

AGARWAL, N.; BREM, A. Frugal and reverse innovation - Literature overview and case study insights from a German MNC in India and China. 18th International Conference on Engineering, Technology and Innovation, 2012.

AGRAWAL, A.; BHATTACHARYA, S.; HASIJA, S. Cost-Reducing Innovation and the Role of Patent Intermediaries in Increasing Market Efficiency. Production and Operations. Management Society, v. 25, n. 2, February, p. 173-191, 2016.

ALBERTI, F. G; PIZZURNO, E. Oops, I did it again! Knowledge leaks in open innovation networks with start-ups. European Journal of Innovation Management, v. 20, n. 1, p. 50-79, 2017.

ANDRADE, A. P. V.; LINS FILHO, M. L.; SILVA, G. G. Capacidade de inovar em startups: Uma abordagem sob a ótica da orientação para a aprendizagem. In: XL Encontro da ANPAD, 2016, Costa do Sauipe. Anais eletrônicos... Costa do Sauípe, 2016. Disponível em< http://www.anpad.org.br/ anpad/abrir_pdf.php?e=MjE1Mjk= >. Acesso em: fev. 2017.

ASSOCIAÇÃO BRASILEIRA DE STARTUPS - ABSTARTUPS. Sobre o CASE: O maior eventos para startups da América Latina, 2014. Disponível em: < https://case.abstartups.com.br/sobre-o-evento-case/ >. Acesso em: maio. 2017.

BARBOSA, R. A.; MACHADO, A. G. C. Estratégias de inovação sob a perspectiva da visão baseada em recursos: um estudo na Embrapa. Gestão \& Regionalidade, v. 29, n. 87, p. 95-110, 2013.

BELKAHLA, W.; TRIKI, A. Customer knowledge enabled innovation capability: proposing a measurement scale. Journal of knowledge management, v. 15, n. 4, p. 648-674, 2011.

BERNE, D. F. O Grau de Inovação das Indústrias MPE da Região Metropolitana Oeste e Sudoeste de São Paulo. Dissertação (Mestrado em Administração). Faculdades Campo Limpo Paulista. São Paulo, 2016.

BESANKO, D.; DRANOVE, D.; SHANLEY, M.; SCHAEFER, S. A Economia da Estratégia. Porto Alegre: Bookman, 2010.

BLEDOW, R.; FRESE, M.; ANDERSON, N.; EREZ, M.; FARR, J. A dialectic perspective on innovation: Conflicting demands, multiple pathways, and ambidexterity. Industrial and Organizational Psychology, v. 2, p. 305-337, 2009.

BRUNSWICKER, S.; VANHAVERBEKE, W. Open innovation in small and medium-sized enterprises (SMEs): external knowledge sourcing strategies and internal organizational facilitators. Journal of Small Business Management, v. 53 n. 4 , p. 1241-1263, 2015.

CAMISÓN, C.; MONFORT-MIR, V. M. Measuring innovation in tourism from the Schumpeterian and the dynamiccapabilities perspective. Tourism Management, v. 33, p. $776-789,2012$.

CARPEJANI, E. A Influência do Programa ALI no processo de inovação de Micro e Pequenas Empresas do Estado de Sergipe. 2015. 115 f. Dissertação (Mestrado em Administração e Desenvolvimento Empresarial) - Universidade Estácio de Sá, Rio de Janeiro, 2015.

CHARLES, R. G.; DAVID, L. Collaborative innovation with customers: A review of the literature and suggestions for future research. International Journal of Management Reviews, v. 14, n. 1, p. 63-84, 2012.

CHESBROUGH, H. Inovação aberta: como criar e lucrar com a tecnologia. Porto Alegre: Bookman, 2012. 241 p.

CHESBROUGH, H. The era of open innovation. MIT Sloan Management Review, v. 44, n. 3, p. 35-41, 2003.

CHRISTENSEN, C. M.; JOHNSON, M. W.; RIGBY, D. K. Foundations for growth: how to identify and build disruptive new business. MIT Sloan Management Review, v.43, n. 3, p. 22-31, 2002.

CROPLEY, D. H.; KAUFMAN, J. C.; CROPLEY, A. J. Measuring Creativity for Innovation Management. Journal of Technology Management \& Innovation, v. 6, n. 3, 2011.

D'ALVANO, L.; HIDALGO, A. Innovation management techniques and development degree of innovation process in service organizations. R and D Management, v. 42, n. 1, 2012.

DAMANPOUR, F.; WALKER, R. M.; AVELLANEDA, C. N. Combinative effects of innovation types and organizational performance: a longitudinal study of service organizations. Journal of Management Studies, v. 46, n. 4, p. 650-675, 2009.

DAVILA, T.; EPSTEIN, M. J.; SHELTON, R. As regras da inovação - como gerenciar, como medir e como lucrar. Porto Alegre: Bookman, 2007.

DOLOREUX, D. What we should know about regional innovation systems of innovation. Technology and Society, v. 24, p. 243-263, 2002.

DRECHSLER, W.; NATTER, M. Understanding a firm's openness decisions in innovation. Journal of Business Research, V. 65, p. $438-445,2012$.

FAUCHART, E.; KEILBACH, M. Testing a model of exploration and exploitation as innovation strategies. Small Business Economics, v. 33, p. 257-272, 2009.

FORSMAN, H. Innovation capacity and innovation development in small enterprises. A comparison between the manufacturing and service sectors. Research Policy, v. 40, p. 739-750, 2011. 
FREEMAN, C.; SOETE, L. A economia da inovação industrial. Campinas: Editora da Unicamp, 2008.

GARCÍA, F.; AVELLA, L.; FERNA'NDEZ, E. Learning from exporting: The moderating effect of technological capabilities. International Business Review, v. 26, n. 6, p. 1099- 1111, 2012.

GILBERT, J. T. Choosing an inovation strategy; theory and practice. Business Horizons, p. 16, nov/dez. 1997.

GOLLO, S.S. Estratégias de Cooperação Competitiva e a Inovação: O caso da Indicação de Procedência Vale dos Vinhedos - RS. 2006, 361 f. Tese (Doutorado em Administração) - Programa de Pós-Graduação em Administração, Universidade Federal do Rio Grande do Sul, Porto Alegre, 2006.

GREER, C. R.; LEI, D. Collaborative Innovation with Customers: A Review of the Literature and Suggestions for Future Research. International Journal of Management Reviews, v.14, n. 1, p.63-84, 2012.

HEIDENREICH, S.; KRAEMER, T. Innovations-Doomed to Fail? Investigating Strategies to Overcome Passive Innovation Resistance. Journal of Product Innovation Management, v. 33, n. 3, p. 277-297, 2016.

HSIEH, C.-T.; HUANG, H.-C.; LEE, W.-L. Using transaction cost economics to explain open innovation in start-ups. Management Decision, v. 54, n. 9, p. 2133-2156, 2016.

HUARNG, K. H.; RIBEIRO-SORIANO, D.E. Developmental management: Theories, methods, and applications in entrepreneurship, innovation, and sense making. Journal of Business Research, v. 67, n. 5, p. 657-662, 2014.

HUNT, R. A. Entrepreneurial tweaking: an empirical study of technology diffusion through secondary inventions and design modifications by start-ups. European Journal of Innovation Management, v. 16, n. 2, p. 148-170, 2013.

IMBUZEIRO, P. E. A. Proposta e Avaliação de um Modelo da Dinâmica da Inovação nas Micro e Pequenas Empresas Atuando em Redes: Um Estudo do Setor de TIC em Alagoas. 2014, 171 f. Tese (Doutorado em Administração) - Programa de Pós-Graduação em Administração, Universidade Federal de Pernambuco. Recife, 2014.

ISMAIL, W. K. W.; ABDMAJID, R. Framework of the culture of innovation: a revisit. Journal Kemanusiaan, v. 9, p. 38-49, 2007.

LENDEL, V.; VARMUS, M. Creation and implementation of the innovation strategy in the enterprise. Economics and management, v. 16, p. 819-825, 2011.

LOVE, J. H.; ROPER, S. SME innovation, exporting and growth: A review of existing evidence. International Small Business Journal, v. 33, n. 1, p. 28-48, 2015.

LUGER, M. I.; KOO, J. Defining and Tracking Businness Strat-Ups. Small Business Economics, v. 24, p. 17-28, 2005.

LYNN, S. G. AKGUN, A. E. Innovation strategies under uncertainty: A contingency approach for new product development. Engineering Management Journal, v. 10, n. 3, p. 11-17, Sept 1998.

MAS-TUR, A.; PINAZO, P.; TUR-PORCAR, A. M.; SÁNCHEZ-MASFERRER, M. What to avoid to succeed as an entrepreneu. Journal of Business Research, v. 68, p. 2279-2284, 2015.

MELO, C.B. B.; NASCIMENTO, J.C. H. B.; MELO, M. A.; BERNARDES J. R.; SOUSA, W. D. Crowdsourcing como uma ferramenta à inovação estratégica empresarial: uma revisão de literatura. Revista de Empreendedorismo, Inovação e Tecnologia, v. 1, n. 1, p. 13-24, 2015.

MIRANDA, J. Q.; SANTOS JUNIOR, C. D.; DIAS, A. T.. A influência das variáveis ambientais e organizacionais no desempenho de startups. Revista de Empreendedorismo e Gestão de Pequenas Empresas, v.5, n.1, 2016.

MOL, M. J.; BIRKINSHAW, J. The sources of management innovation: When firms introduce new management practices. Journal of Business Research, p.1-13, 2009.

OECD - Organization for Economic Co-operation and Development, Manual de Oslo - Diretrizes para coleta e interpretação de dados sobre inovação, OECD - tradução FINEP, Brasília, 2006.

PARK, S.; STYLIANOU, A.; SUBRAMANIAM, C.; NIU, Y. Information technology and interorganizational learning: An investigation of knowledge exploration and exploitation processes. Information \& Management, v. 52, p. 998-1011, 2015.

PÉREZ-LUÑO, A.; MEDINA, C.C.; LAVADO, A.C.; RODRÍGUEZ, G.C. How social capital and knowledge affect innovation. J. Bus. Res, v.64, n. 12, p. 1369-1376, 2011.

RADJOU, N.; PRABHU, J. Frugal Innovation: A New Business Paradigm. 2013. Disponível em: <http://knowledge.insead.edu/innovation/frugal-innovation-a newbusiness-paradigm-2375>. Acessado em: março de 2017.

RAMADANI, V.; S. GERGURI. Theoretical Framework of innovation and competitiveness and innovation program in Macedonia. European Journal of Social Sciences, v. 23, n. 2, p. 268-276, 2011.

REN, S.; EISINGERICH, A. B.; TSAI, H.-T. How do marketing, research and development capabilities, and degree of internationalization synergistically affect the innovation performance of small and medium-sized enterprises (SMEs)? A panel data study of Chinese SMEs. International Business Review, v. 24, p. 642-651, 2015. 
RIES, E. A Startup Enxuta: como os empreendedores atuais utilizam a inovação contínua para criar empresas extremamente bem-sucedidas. Lua de Papel, $1^{\text {a }}$ edição. São Paulo-SP, 2012.

ROBEHMED, N. What is a Startup? Forbes. Disponível em: <www.forbes.com/sites/natalierobehmed/2013/12/16/what-isastartup/\#5c77b0c64c63> Acesso em: março. 2017.

SAEBI, T.; FOSS, N. J. Business models for open innovation: matching heterogenous open innovation strategies with business model dimensions. Center for Service Innovation-Department of Strategy and Management Norwegian School of Economics, 45 f. 2014.

SANCHES, P. L. B.; MACHADO, A. G. C. Estratégias de inovação sob a perspectiva da Resourced-Based View: análise e evidências em empresas de base tecnológica. Gest. Prod., São Carlos, v. 21, n. 1, p. 125-141, 2014.

SCHUMPETER, J. The Theory of Economic Development. Harvard University Press, Cambridge Massachusetts, 1939.

SCHUMPETER, J.A. A teoria do desenvolvimento econômico. São Paulo: Nova Cultural, 1988.

SCHUMPETER, J. A. Teoria do desenvolvimento econômico: uma investigação sobre lucros, capital, crédito, juro e o ciclo econômico (1 ed., 1934). Tradução de Maria Sílvia Possas. Coleção Os Economistas. São Paulo: Nova Cultural, 1998.

SEBRAE. 5 startups de tecnologia para ficar de olho, 2015. Disponível em: < http://startupsebraeminas.com.br/5-startupsde-tecnologia-para-ficar-de-olho/ >. Acesso em Jun. 2017.

SERAN, S.; IZVERCIAN, M.. Prosumer engagement in innovation strategies The Prosumer Creativity and Focus Model. Management Decision, v. 52, n. 10, p. 1968-1980, 2014.

SHONTELL, A. This is the definitive definition of a startup", Business Insider UK, 31 December 2014. Disponível em: <http://uk.businessinsider.com/what-is-a-startup-definition-2014-12?r=US\&IR=T>. Acesso em: Feb. 2017

SILVA, S. M.; WESCHENFELDER, C. F.; ESTEVES, P. C. L. Avaliação da capacidade de inovação das empresas do setor de tecnologia da informação de um município da região do extremo sul de Santa Catarina. In: Seminário de parques tecnológicos e incubadoras de empresa, 2014, Passo Fundo. Anais eletrônicos... Passo Fundo, 2014. Disponível em: < http://www.anprotec.org.br/Relata/ArtigosCompletos/ID\%2091.pdf >. Acesso em: Março. 2017.

STOILOV, I. A. Innovation in technological start-ups: Korean start-up ecosystem. Universitat Autonoma de Barcelona Degree: Business Administration and Management, 2015.

TIDD, J.; BESSANT, J.; PAVITT, K. Gestão da inovação. Porto Alegre: Bookman, 2008.

TIWARI, R.; KALOGERAKIS, K..; HERSTATT, C. Frugal innovation and analogies: some propositions for product development in emerging economies. Working Paper, n. 84, Hamburg University of Technology, v. 49, p, 15-23, 2014.

TURRI, S. N. Z. WAGNER, B. S. Fatores críticos de sucesso de startups/TI. In: IV Simpósio Internacional de Projetos, Inovação e Sustentabilidade, 2015, São Paulo. Anais... São Paulo, 2015. Disponível em: < https://singep.org.br/4singep/resultado/280.pdf>. Acesso: abr. 2017.

VARRICHIO, P. C.. Uma discussão sobre a estratégia de inovação aberta em grandes empresas e os programas de relacionamento voltados para startups no Brasil. RACEF - Revista de Administração, Contabilidade e Economia da Fundace. v. 7, n. 1, Ed. Esp. Ecossistemas de Inovação e Empreendedorismo, p. 148-161, 2016.

VERLEYE, K. The co-creation experience from the customer perspective: its measurement and determinants. Journal of Service Management, v. 26 n. 2, p. 321-342, 2015. 